Documentation et bibliothèques

\title{
Le parcours exigeant et inusité d'un bâtisseur : Georges Cartier (1929-1994), fondateur de la Bibliothèque nationale du Québec
}

\section{Céline Robitaille-Cartier}

Volume 51, numéro 3, juillet-septembre 2005

URI : https://id.erudit.org/iderudit/1029499ar

DOI : https://doi.org/10.7202/1029499ar

Aller au sommaire du numéro

Éditeur(s)

Association pour l'avancement des sciences et des techniques de la

documentation (ASTED)

ISSN

0315-2340 (imprimé)

2291-8949 (numérique)

Découvrir la revue

Citer ce document

Robitaille-Cartier, C. (2005). Le parcours exigeant et inusité d'un bâtisseur :

Georges Cartier (1929-1994), fondateur de la Bibliothèque nationale du Québec.

Documentation et bibliothèques, 51(3), 203-206.

https://doi.org/10.7202/1029499ar

Tous droits réservés (c) Association pour l'avancement des sciences et des techniques de la documentation (ASTED), 2005
Ce document est protégé par la loi sur le droit d'auteur. L'utilisation des services d’Érudit (y compris la reproduction) est assujettie à sa politique d'utilisation que vous pouvez consulter en ligne.

https://apropos.erudit.org/fr/usagers/politique-dutilisation/ 


\title{
Le parcours exigeant et inusité d'un bâtisseur: Georges Cartier (1929- 1994), fondateur de la Bibliothèque nationale du québec
}

\author{
CÉLINE ROBITAILLE-CARTIER* \\ ccartier2@hotmail.com
}

Allocution présentée le 18 novembre 2004 à l'occasion de la remise, pour une première fois, de la bourse GeorgesCartier à $M^{m e}$ Stéphanie Smith, étudiante à l'École de bibliothéconomie et des sciences de l'information (EBSI). Après avoir félicité la lauréate et remercié l'EBSI, $M^{m e}$ Céline Robitaille-Cartier résume la carrière professionnelle de Georges Cartier.

$\mathrm{N}$ ous AVONS PASSÉ, GEORges CARTIER et moimême, plus de trente années dans l'univers des bibliothèques; notre engagement dans le développement de la bibliothéconomie et des sciences de l'information, dans l'évolution de la profession de bibliothécaire, dans la recherche de l'excellence a été constante et, surtout, passionnante.

Il est toujours un peu malaisé de parler objectivement de quelqu'un dont on a partagé la vie durant quarante années, quelqu'un qu'on a accompagné, aimé et admiré. Je vais toutefois tenter de décrire brièvement le cheminement professionnel assez singulier de Georges Cartier. En même temps, ce sera un aperçu de cette période lointaine des années 1960, que la plupart d'entre vous n'ont pas connue, celle qui a précédé la création de la Bibliothèque nationale du Québec et sa fondation.

Après des études classiques et avoir poursuivi, depuis sa prime enfance, la pratique intensive $d u$ piano, Georges Cartier est éligible au prix Archambault qui lui permettrait de poursuivre des études musicales, mais il rêve aussi de devenir écrivain. Un projet, pour le moins insolite, lui est proposé à sa sortie du collège par un inconnu qui se recommande $d u$ professeur de littérature du Collège de l'Assomption: écrire un roman ayant pour thème «la vie d'une jeune fille handicapée » que l'individu souhaitait publier sous son nom. Cet événement étrange apparut à Georges Cartier comme un «signe», un présage. Il accepta le projet et s'y consacra durant un an. Il dira plus tard que cet épisode lui a permis de "fouiller» la langue française et aura été déterminant dans son choix de carrière. Une fois terminé ce travail de nègre, comme on dit dans le milieu littéraire, il s'inscrit à la Faculté des lettres de l'Université de Montréal où il obtient une

* Céline Robitaille-Cartier a été directrice générale des bibliothèques de l'Université Laval de 1978 à 1989. licence en littérature et études gréco-latines. Après une scolarité de doctorat, il s'inscrit à l'École de bibliothécaires où il obtient le premier prix d'excellence. À la sortie de l'École, il travaille durant quelques années à la Commission des écoles catholiques de Montréal, au moment où le réseau des bibliothèques scolaires est mis en place. Il fait paraître pendant ces années quatre recueils de poèmes, Hymnes-Isabelle, Laves et Neiges, Obscure navigation du temps et La Mort à vivre, publié en Belgique, qui lui valut le prix Interfrance.

À la fin des années 1950, le Québec vivait toujours sous le régime duplessiste, sans horizon, sans ouverture sur le monde extérieur. (Il n'y avait alors à Montréal à peu près pas de théâtre ni de cinéma intéressant. Il fallait aller à New York ou à Boston pour voir une grande exposition d'art.) Nous avons décidé à l'automne de 1957 de quitter le Québec pour aller vivre en France, ce pays que nous ne connaissions que de façon livresque. Nous sommes donc partis sur un modeste bateau, avec notre petite fille de deux ans, trois valises et une machine à écrire. La rédaction de textes littéraires pour Radio-Canada, une bonne soixantaine, nous apportait quelques ressources financières. Ce séjour nous a permis de voyager en France et en Italie, d'élargir notre culture, de visiter des musées, de connaître le théâtre. Il permit aussi à Georges Cartier de rédiger le premier jet d'un deuxième roman qui sera réécrit dans les années suivantes et publié en 1964 sous le titre Le Poisson pêché. Ce roman fut couronné par le prix du Cercle du livre de France.

À l'été de 1958, après la relâche des émissions culturelles de Radio-Canada, les vivres vinrent à manquer et nous sommes revenus au Québec. Georges Cartier fut presque immédiatement engagé par le Collège Sainte-Marie qui lui donna le mandat d'organiser et de développer la bibliothèque qui allait plus tard servir d'assise à la future bibliothèque de l'UQAM. En 196o, 


\section{Nous sommes donc revenus au Québec au début de 1964. Georges Cartier, après la publication $d u$ Poisson pêché, dut mettre en veilleuse, et pour plusieurs années, sa carrière d'écrivain.}

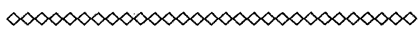

le recteur de l'Université de Montréal lui demandait d'analyser la situation de l'École de bibliothécaires et de lui présenter un rapport pour donner à cette école une dimension vraiment universitaire. La même année, l'Unesco tenait à Montréal une conférence sur l'éducation des adultes. Georges Cartier accepta d'organiser une salle de presse et de mettre en place les mécanismes de communication avec les médias. À la fin de la conférence le directeur de l'information de l'Unesco, ravi de la couverture médiatique de la rencontre (à Paris, avouait-il, les médias n'avaient guère d'intérêt pour les activités de l'Unesco), lui offrait de venir à Paris mettre sur pied un service de distribution de l'information aux quelque 100 pays membres de l'organisation et de créer un centre de documentation pour les correspondants étrangers. Et c'est ainsi que nous repartions pour l'Europe. Nous y sommes demeurés un peu plus de trois ans.

À notre arrivée à Paris, l'Unesco venait de lancer une campagne mondiale de financement (mars 1960) pour sauver les 24 temples égyptiens qui menaçaient d'être engloutis par la construction du haut barrage d'Assouan (il est inutile de dire que la diffusion de l'information relative à la campagne - description du projet et des monuments, évolution des entrées de fonds, plans de sauvetage proposés, communiqués de presse, entrevues - fut l'une des priorités du service de distribution de l'information). Au cours de ce deuxième séjour, avec un salaire assuré, cette fois, c'est toute l'Europe qui nous était offerte. Nous avons pu visiter, entre autres, les bibliothèques nationales d'une bonne dizaine de pays.

En 1962, Georges Cartier étant de passage à Montréal à l'occasion du décès de son père, le sousministre des Affaires culturelles, M. Guy Frégault, lui demande de rentrer au Québec pour prendre la direction de la Bibliothèque Saint-Sulpice, et «c'était urgent!» insistait-il. Il souhaitait redonner vie à cette belle et riche institution en état d'hibernation, dont le ministère venait d'hériter du Secrétariat de la province. L'établissement avait été fermé de 1931 à 1944, date où le gouvernement du Québec en avait fait l'acquisition. Il n'est pas exagéré de dire que cette bibliothèque avait stagné pendant une trentaine d'années et que l'édifice qui l'abritait était en très mauvais état. (Les journalistes l'appelaient le «sarcophage des Sulpiciens»). «Cette bibliothèque ne remplissait aucune fonction particulière, écrira Cartier, et, le moins qu'on puisse dire, avait été fort négligée au fil des années et à tous points de vue: immeuble mal entretenu, conditions déplorables de conservation et d'organisation des collections, personnel très réduit et très peu motivé, moyens financiers nettement insuffisants». Il refusa l'invitation qui lui était faite parce que, d'une part, le poste n'était guère alléchant et, d'autre part, parce que l'implantation des projets à l'Unesco exigerait encore au moins huit mois de travail. Il était hors de question de ne pas respecter ses engagements à l'égard de l'Unesco.

Au printemps de 1963, M. Frégault, de passage à Paris pour inaugurer une exposition de peintres québécois, le contacta de nouveau (aucun bibliothécaire digne de ce nom n'avait-il pas entre-temps accepté la direction de la Bibliothèque Saint-Sulpice...?). La rencontre fut décisive: Georges Cartier accepta de revenir au Québec et de prendre la direction de Saint-Sulpice à condition "qu'on dote l'institution d'un statut particulier... et de ressources adéquates». "Là, d'un commun accord, nous avons fait le projet de doter le Québec de sa propre Bibliothèque nationale. La Bibliothèque Saint-Sulpice servirait d'assise", rappellera Cartier dans un document publié à l'occasion du $25^{\mathrm{e}}$ anniversaire de la BNQ. Cette entente "conclue à huis clos", plusieurs mois de correspondance s'écoulèrent avant que l'accord ne soit scellé. Il fallait négocier les conditions d'acceptation du poste, en particulier la restauration du «mausolée» de la rue Saint-Denis (ministère des Travaux publics) et l'attribution de ressources adéquates pour transformer la Bibliothèque Saint-Sulpice en Bibliothèque nationale (ministère des Finances et fonction publique). Assuré de l'appui du sous-ministre des Affaires culturelles, et anticipant le support des milieux culturels, Georges Cartier remit son avis de départ à l'Unesco. Il avait beaucoup réfléchi à cette offre du sous-ministre au cours des mois précédents. En acceptant de relever un défi plutôt périlleux et certainement téméraire, il comptait sur le soutien de ses collègues bibliothécaires et croyait que "le contexte d'effervescence nationaliste au Québec qu'on a qualifiée de "Révolution tranquille" favoriserait le projet de création d'une bibliothèque nationale pour les Québécois. Le Québec sortait d'une ère marquée par la stagnation sociale et politique, et dominée par un clergé tout-puissant», écrira-t-il. "L'avènement au pouvoir des libéraux, dirigés par Jean Lesage, soulevait un espoir de renouveau dans tous les domaines et le Québec s'appropriait les clés de sa destinée en se dotant d'institutions propres à assurer son plein développement», écrira-t-il encore.

Nous sommes donc revenus au Québec au début de 1964. Georges Cartier, après la publication du Poisson pêché, dut mettre en veilleuse, et pour plusieurs années, sa carrière d'écrivain. Il écrira épisodiquement des textes littéraires pour Radio-Canada, 
en particulier pour L'Atelier des inédits, et une pièce de théâtre. Les années suivantes furent consacrées à la préparation de la loi 91, à la réfection du bâtiment de la rue Saint-Denis (il y eut pour plus de 1 demi-million de dollars de travaux), au recrutement du personnel, qui est passé d'une quinzaine d'employés à plus de 80 et, surtout, à la sensibilisation des milieux culturels dont il fallait obtenir l'appui pour faire éclore le projet de bibliothèque nationale. Il eut plusieurs rencontres avec les associations de bibliothécaires, l'ACBLF (Association canadienne des bibliothécaires de langue française, l'ancêtre de l'ASTED), et la Corporation des bibliothécaires professionnels s'infiltra dans les regroupements d'écrivains, de libraires et d'éditeurs pour «semer» l'idée d'une bibliothèque nationale. À peu près personne, à l'époque, en dehors du milieu professionnel, malgré la création de la Bibliothèque nationale du Canada à Ottawa en 1953, n'avait la moindre idée de ce qui pouvait caractériser une bibliothèque dite "nationale». On parlait surtout d'une «bibliothèque provinciale» (ce qui n'est qu'un titre de propriété), puis de bibliothèque d'État (ce qui n'est guère plus significatif), pendant que Georges Cartier discutait de «bibliothèque nationale» avec les services juridiques du ministère des Affaires culturelles que le qualificatif «nationale» effrayait au plus haut point. Il réussit à convaincre les autorités du ministère que, dans le vocabulaire bibliothéconomique, et ce partout dans le monde, le mot nationale n'avait pas de connotation politique et qualifiait essentiellement le statut d'une institution "gardienne du patrimoine documentaire d'une nation». Cet adjectif n'avait encore jamais été utilisé pour aucune institution gouvernementale (l'Assemblée nationale était alors «législative»). C'est l'ACBLF, lors d'une assemblée générale en octobre 1964, qui donnera le coup d'envoi en formulant le vœu (encore bien timide) d'étudier la transformation de la Bibliothèque Saint-Sulpice en bibliothèque provinciale. À l'été 1965, Georges Cartier préside un comité de l'ACBLF qui, dans son rapport, recommande que soit créée une bibliothèque d'État ayant les fonctions d'une bibliothèque nationale. Le ministre Pierre Laporte reprendra ce projet dans son livre blanc.

Il a fallu trois ans de discussions, de pourparlers, de rencontres, d'échanges et de consultations avant que le projet de loi ne soit finalisé, et le ministre s'apprêtait à le présenter à l'Assemblée législative lorsque les libéraux furent défaits aux élections de 1966. C'est donc sous le gouvernement de l'Union nationale que la loi fut sanctionnée, et à l'unanimité. Il fallait désormais mettre en place des structures prévues dans la loi - dépôt légal, bibliographie nationale courante, mécanismes de collaboration avec les bibliothèques du Québec et de l'étranger -, prévoir l'automatisation des services, établir un plan de construction, l'espace étant déjà limité, malgré l'achat de la Jewish Public Library, rue de l'Esplanade, rebaptisée annexe
Son mandat de directeur étant terminé, il acceptait, à la demande du ministre des Affaires culturelles, de mettre sur pied une direction générale des arts et des lettres.

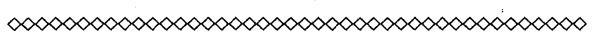

Ægidius-Fauteux en l'honneur du premier conservateur de la Bibliothèque Saint-Sulpice.

En 1973, survint ce que les journaux ont appelé «l'Affaire des manuscrits» qui éclata après la divulgation d'une directive ministérielle ordonnant au conservateur en chef «de transférer tous les manuscrits littéraires et culturels, y compris les cartes et plans non publiés, les photographies originales et les microfilms de documents non publiés aux Archives de la province», ce qui impliquait la cession aussi bien des lettres des patriotes de 1837 que les manuscrits des auteurs modernes et contemporains, tels Nelligan, Aquin, Miron, Lalonde et combien d'autres, versés massivement à la Bibliothèque nationale durant les événements d'octobre 1970. Cette directive suscita une crise majeure et les protestations virulentes des associations de bibliothécaires, d'éditeurs, d'écrivains et des chercheurs de toutes disciplines. La querelle entraîna aussi la démission du conservateur qui avait tenté en vain, pendant sept mois, de dissuader les autorités du ministère de mettre cette directive en application, et qui ne pouvait être solidaire d'une politique dénoncée publiquement par tous les intéressés (la plupart des écrivains qui comptent en ce pays, un grand nombre de chercheurs, etc.), comme l'écrivait Jacques Hébert dans la préface de «l'Affaire des manuscrits», directive considérée, à cette époque, «aussi aberrante qu'absurde». Quelques mois après sa démission, Georges Cartier devenait professeur à l'EBSI, puis directeur de l'École pour les quatre années suivantes (1973-1977).

Son mandat de directeur étant terminé, il acceptait, à la demande du ministre des Affaires culturelles, de mettre sur pied une direction générale des arts et des lettres. Stimulé par ce nouveau défi, il se disait qu'après s'être préoccupé de la santé et de l'éducation, le gouvernement allait enfin s'intéresser à la culture...! Par la suite, deux années de recherche à la Mission gouvernementale permanente, à laquelle l'ÉNAP participait, permirent à Georges Cartier de publier un essai sur Les Relations culturelles internationales du Québec et d'étudier, à la demande du cabinet du premier ministre, l'impact du libre-échange sur les activités culturelles québécoises. En 1986, le ministre des Affaires culturelles lui proposa de reprendre la direction de la Bibliothèque nationale pour y préparer une nouvelle loi qui allait faire de l'institution une société d'État, c'est-à-dire un organisme disposant d'une autonomie plus grande. Il mit trois ans à réaliser 


\section{L'Université de Moncton lui avait décerné un doctorat honoris causa} pour «sa contribution à l'épanouissement de la vie culturelle et la mise en valeur du patrimoine québécois et son rayonnement à l'étranger ».

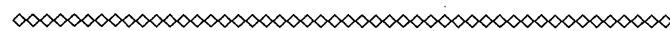

ce mandat (ce qui correspondait à léchéancier qu'il avait proposé à $\mathrm{M}^{\mathrm{me}}$ Lise Bacon) et, malgré l'insistance de la ministre pour qu'il accepte le mandat de pdg de la nouvelle corporation, il prit sa retraite en 1989. L'année précédente, l'Université de Moncton lui avait décerné un doctorat honoris causa pour «sa contribution à l'épanouissement de la vie culturelle et la mise en valeur du patrimoine québécois et son rayonnement à l'étranger».

À partir de 1976 et jusqu'à son décès, en 1994, Georges Cartier s'était remis à l'écriture de façon inter- mittente et a publié un nouveau roman, Notre-Dame$d u$-Colportage, une rétrospective de sa poésie intitulée Chanteaux, et deux récits: L'Odyssée intime de Jacques Cartier et Dans les fougères de l'enfance. Un recueil de poèmes posthumes, Au delta de l'âme, a aussi paru en 2001. Un dernier roman, Soir de cendres, écrit au cours des deux dernières années de sa vie, porte la mention (qui, d'évidence, m'est adressée): "Ne pas publier si je n'ai pas eu le temps de réviser le texte avant de mourir.» Il se savait en sursis depuis 1989.

Voilà donc, la synthèse du parcours de celui que la remise d'une bourse éponyme veut honorer, à l'occasion du dixième anniversaire de son décès. Ce parcours, interrompu trop tôt, a été des plus heureux et passionné, malgré quelques inévitables antagonismes et épreuves survenus en cours de route. Personnellement, j'ai été comblée de le partager. Puisse ce cheminement professionnel insuffler chez les étudiants et les étudiantes de l'EBSI le désir de relever les défis les plus exigeants! -1

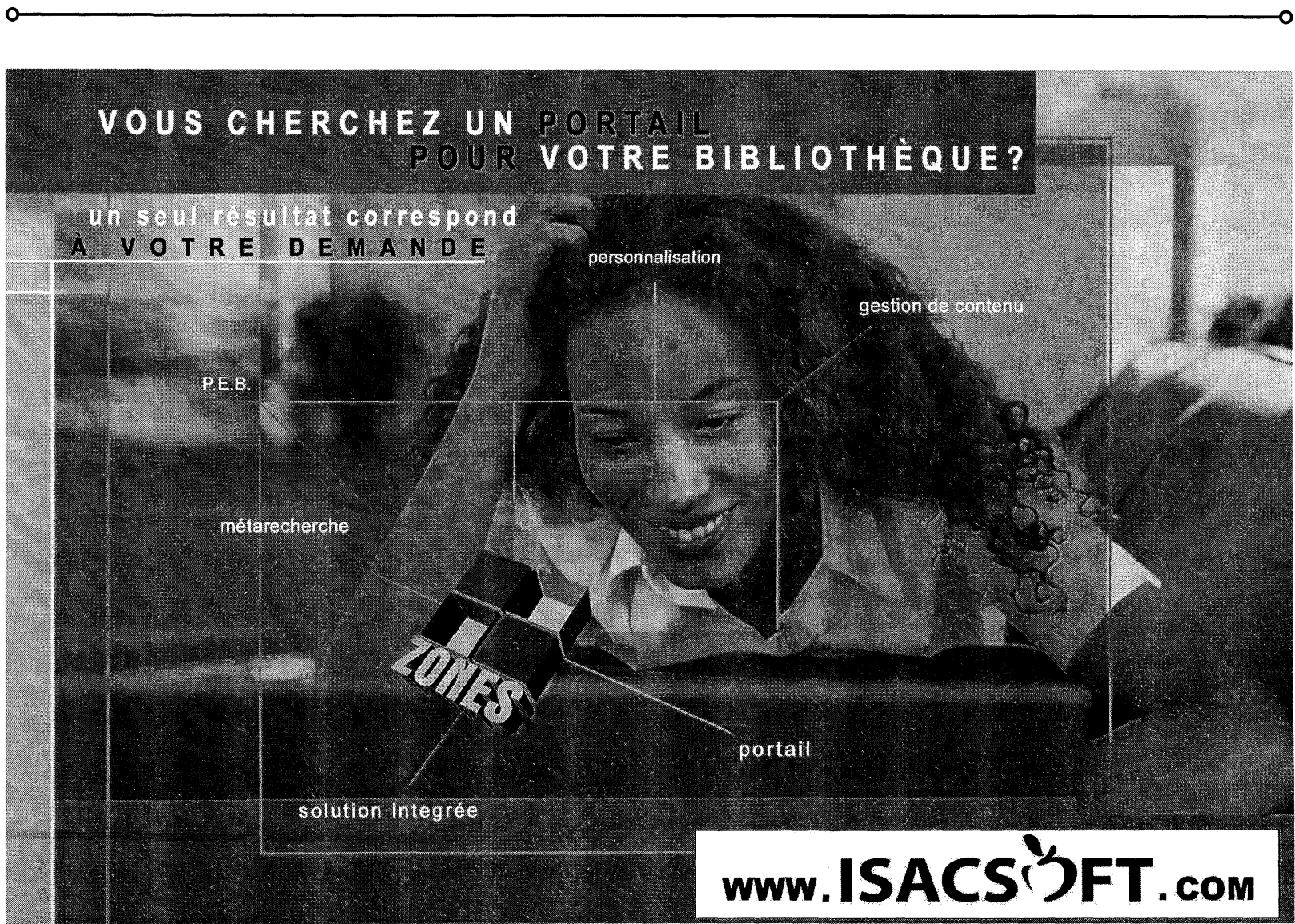

